\title{
Experience with radiofrequency catheter ablation of fascicular tachycardia
}

\author{
David H Bennett
}

\begin{abstract}
Objective-To report experience with radiofrequency catheter ablation of fascicular tachycardia including two cases of the rare type of this arrhythmia which arises from the anterior fascicle of the left bundle branch.

Design-Review of results of radiofrequency ablation in nine consecutive patients presenting with fascicular tachycardia.

Setting-Regional cardiac centre.

Intervention-Percutaneous radiofrequency catheter ablation, performed between 1993 and 1996.

Results-Radiofrequency ablation was successful in both patients with tachycardia arising from the anterior fascicle and in six of the seven patients with tachycardias arising from the posterior fascicle. Notable differences in the "right bundle branch block" configuration of lead V1 during tachycardia between patients were observed. One patient with incessant tachycardia had marked impairment of ventricular function which returned to normal after ablation.

Conclusions-Radiofrequency ablation is effective in both anterior and posterior fascicular tachycardias. The arrhythmia can cause reversible impairment of ventricular function.
\end{abstract}

(Heart 1997;77:104-107)

Keywords: radiofrequency ablation; fascicular ventricular tachycardia; tachycardia

Fascicular tachycardia is thought to arise from Purkinje fibres in either the posterior fascicle or, rarely, in the anterior fascicle of the left bundle branch. It usually occurs in young people without structural heart disease. A posterior fascicular origin results in ventricular complexes during tachycardia with a right bundle branch block and left axis configuration while an anterior fascicular origin leads to

University Department of Cardiology, Regional Cardiac Centre, Wythenshawe Hospital, Manchester D H Bennett D H Bennett

Correspondence to: Dr D Bennett, Department of Cardiology, Wythenshawe Hospital, Manchester M23 9LT.

Accepted for publication 16 July 1996 patient undergoing ablation for idiopathic tachycardia arising from the anterior fascicle. ${ }^{2-6}$ Results of radiofrequency catheter ablation and some clinical details in nine consecutive cases of fascicular tachycardia, including two with right bundle branch block and right axis deviation morphology, are reported in this paper.

\section{Patients and methods}

Nine patients with fascicular tachycardia (aged 21-48; six men) underwent radiofrequency catheter ablation between October 1993 and May 1996. Their history of paroxysmal tachycardia ranged from two to 25 years. Seven had been referred with a diagnosis of supraventricular tachycardia. Antiarrhythmic drugs had failed to prevent tachycardia in all patients: five had received verapamil, five flecainide, and four sotalol.

All patients had normal findings on clinical examination and normal electrocardiograms during sinus rhythm. One patient, who had incessant tachycardia at a rate of 140-160 beats per minute, had an abnormal echocardiogram: left ventricular function was markedly impaired with an estimated ejection fraction of $30 \%$. One of the patients whose arrhythmia arose from the anterior fascicle had been shown by ambulatory electrocardiography to have 21000 and 39000 unifocal ventricular extrasystoles in two 24 hour periods, including long periods of bigeminy and frequent interpolated beats.

Each patient underwent an electrophysiological study and radiofrequency catheter ablation. Antiarrhythmic drugs were stopped at least 48 hours before. Informed written consent was obtained.

Quadripolar electrode catheters were advanced under local anaesthesia via the right femoral vein to the right atrium and to the right ventricle, across the tricuspid valve and, in one case, to the coronary sinus. Left ventricular endocardial mapping was carried out using a $7 \mathrm{~F}$ steerable quadripolar electrode with a $4 \mathrm{~mm}$ tip which was advanced from the right femoral artery. Intracardiac electrograms were displayed with recordings from the limb leads and lead V1. A separate electrocardiograph was used to record the full 12 lead electrocardiogram.

Tachycardia was initiated by programmed premature ventricular stimulation, or by overdrive atrial or ventricular pacing. If ventricular tachycardia could not be induced, intravenous isoprenaline was given.

The site for ablation was sought by locating the earliest onset of ventricular activation and, in five patients, by pace mapping. Radiofrequency energy generated using a Medtronic Atakr system was delivered via the $4 \mathrm{~mm}$ tip of 
Figure 1 Left posterior fascicular tachycardia. Leads $a V L, I I, a V F$, and electrograms from mapping (MAP) and His bundle (HB) electrodes. $A$ fascicular potential precedes the ventricular electrogram recorded from the mapping electrode.

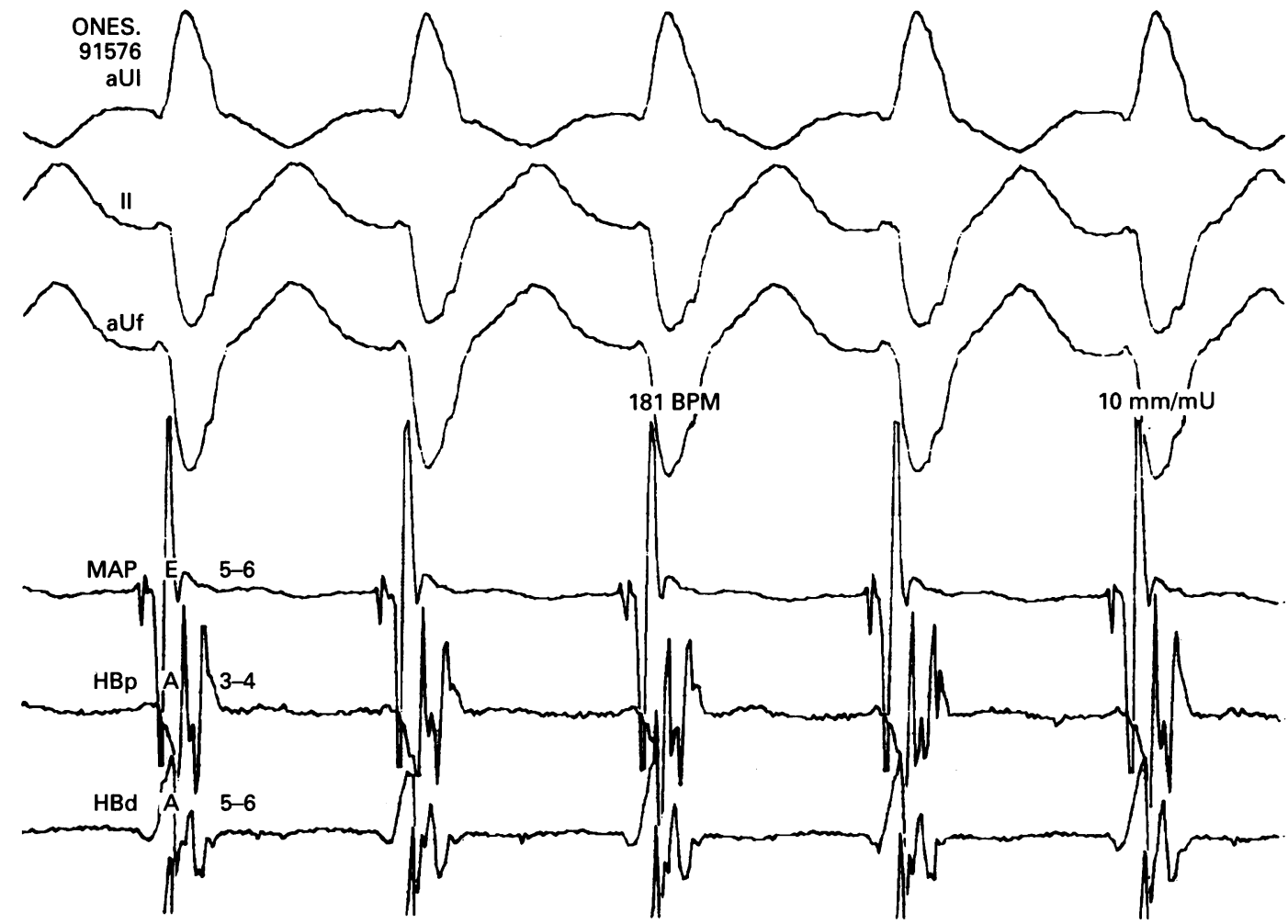

the mapping electrode. A large electrode applied to the skin over the left scapula acted as the indifferent pole.

Two patients who had had infrequent episodes of tachycardia underwent a brief pacing study at a later date to see if the arrhythmia was still inducible.

\section{Results}

\section{SURFACE ELECTROCARDIOGRAM DURING} TACHYCARDIA

The heart rate ranged from 140 to 220 beats per minute. There was notable variation in morphology of lead V1 during tachycardia. In only two cases was the appearance of lead V1 absolutely typical of right bundle branch block: in two patients there was a $Q$ wave at the onset of the complex and in five patients there was a wide notched $R$ wave without an $S$ wave.

QRS duration ranged from $0 \cdot 11$ to $0 \cdot 14 \mathrm{~s}$. There was left axis deviation (ranging from -60 to -120 degrees) in seven patients and right axis deviation (+120 degrees) in two patients. The surface electrocardiogram showed atrioventricular dissociation in six patients: independent $\mathbf{P}$ waves in three patients and capture or fusion beats in three patients.

\section{ELECTROPHYSIOLOGICAL PROCEDURE}

Tachycardia was induced by programmed or overdrive ventricular pacing in three patients and by rapid atrial pacing in three patients. There was spontaneous tachycardia in three patients, in one only during isoprenaline infusion.

In each case, there was a brief potential immediately before the ventricular electrogram at the site of earliest activation, which preceded the onset of the surface ECG QRS complex by $20-35 \mathrm{~ms}$ (figs 1 and 2). Pace mapping resulted in identical ventricular complexes in 11 of the ECG leads in three patients, 10 leads in one patient, and seven leads in another patient.

In the patients with left posterior fascicular tachycardia, the origin of the tachycardia was located to the region of the posteroapical left ventricular septum.

In the patients with right axis deviation during tachycardia, earliest activation, preceded by a sharp potential, was found in the anterior lateral wall of the left ventricle, about $2 \mathrm{~cm}$ inferior to the aortic valve. Figures 2 and 3 show the surface and intracardiac electrograms, and results of pace mapping in one of these patients: there was no evidence of an accessory atrioventricular pathway and in particular, atrioventricular dissociation was observed at times during tachycardia.

In all cases, tachycardia was not inducible at the end of the procedure, even during infusion of isoprenaline. There were no complications.

In one patient who had undergone an apparently successful ablation, tachycardia was subsequently induced using a previously implanted antitachycardia pacemaker. There had been problems at ablation with the recording system and difficulty initiating tachycardia but the arrhythmia had not been inducible after radiofrequency energy had been delivered to a site of early ventricular activation preceded by a sharp potential. There have been no recurrences of tachycardia in any of the other patients. Fascicular tachycardia was not inducible in the other patient who underwent a repeat pacing study.

Repeat ambulatory electrocardiography in the patient who had had extremely frequent extrasystoles before ablation demonstrated only nine ventricular extrasystoles during a 24 hour period. 
Figure 2 Left anterior fascicular tachycardia. Leads I, II, III, aVR, $a V F, V 1$, and electrograms recorded from the mapping $(M A P)$, right atrial $(R A)$, His bundle (HBE), right ventricular apex (RVA), and coronary sinus (CS) electrodes. Arrow points to fascicular electrogram.

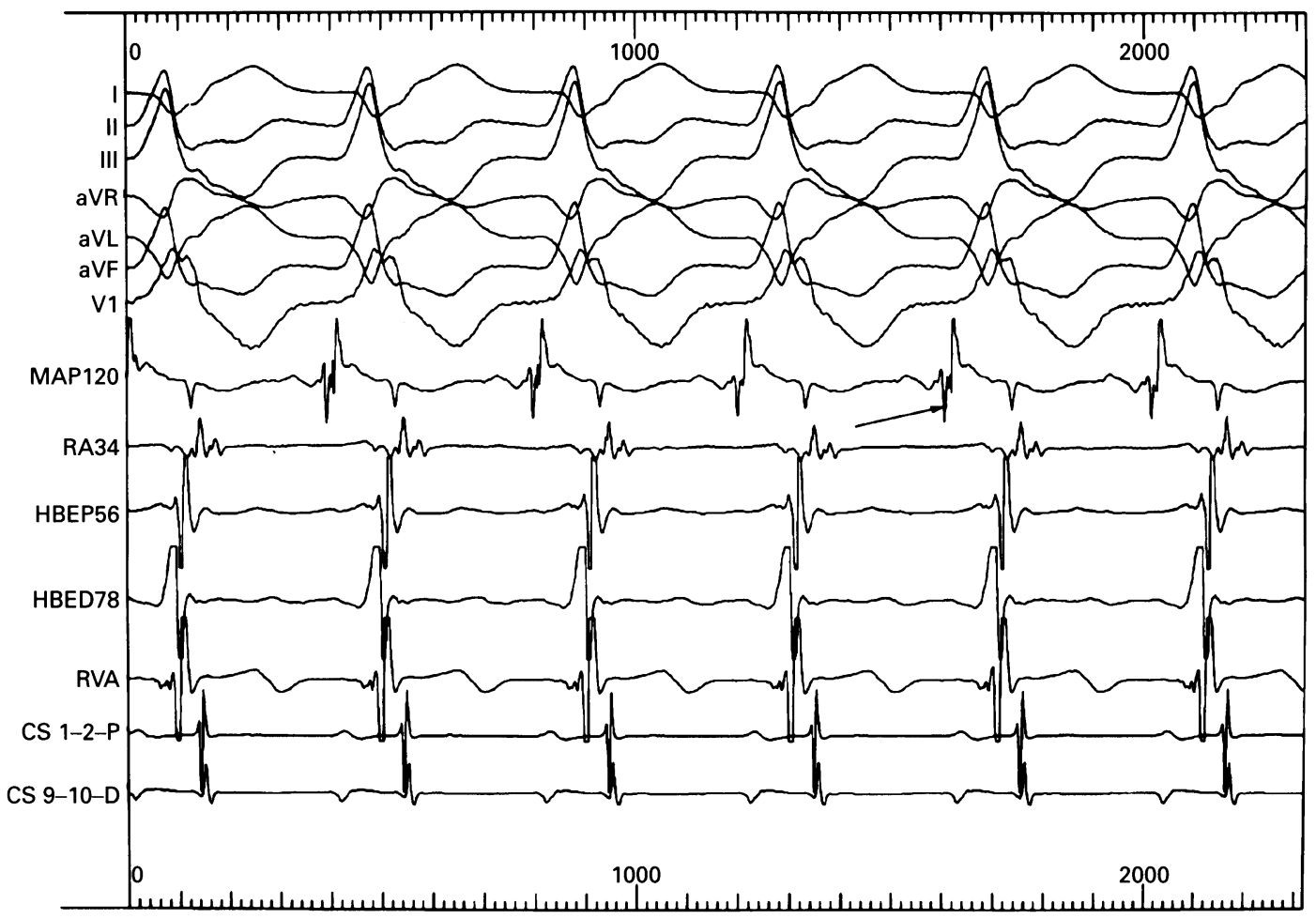

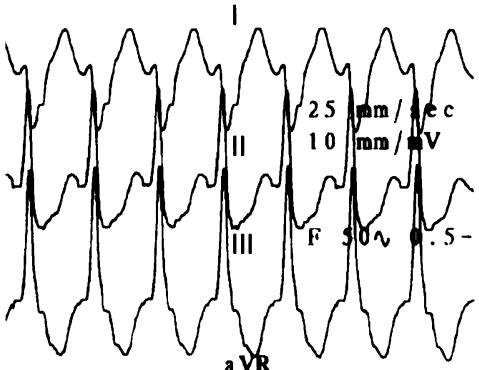
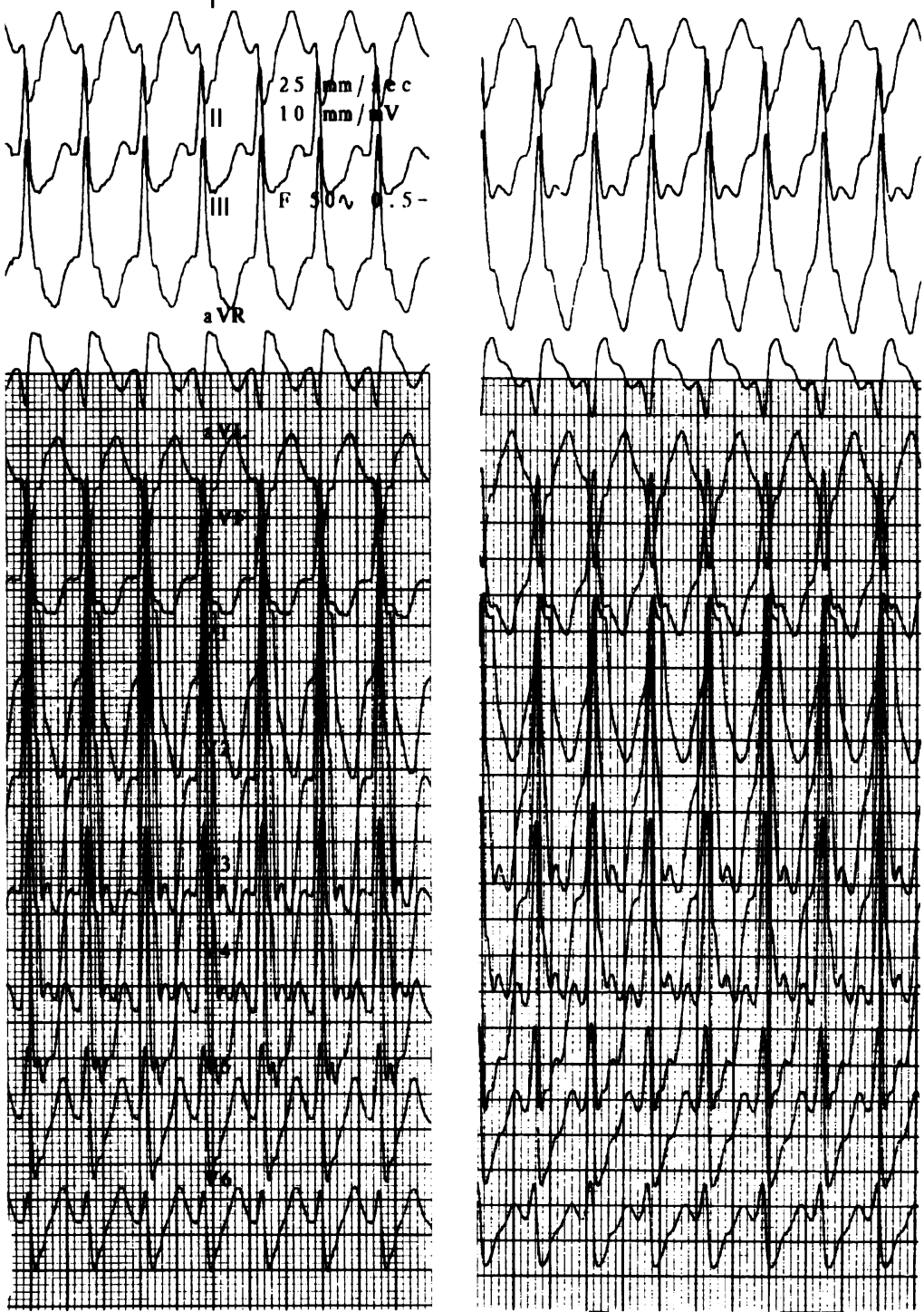

Figure 3 Pace mapping in a patient with left anterior fascicular tachycardia. 12 lead electrocardiograms during pacing via mapping electrode at site of recording of fascicular potential (left-hand panel) and during fascicular tachycardia (right-hand panel) were very similar.

\section{Discussion}

As others have found, tachycardia could be initiated by premature or overdrive ventricular stimulation in some patients while only atrial overdrive pacing was successful in others.? Differing methods of initiation of tachycardia point to more than one mechanism being responsible for fascicular tachycardias, ${ }^{8}$ as does the demonstration in one of the patients with anterior fascicular tachycardia of extremely frequent ventricular extrasystoles.

In each case, the ventricular electrogram at the site of successful ablation was preceded by a brief potential as described by Nakagawa $e t$ $a l$ in their eight cases of tachycardia originating from the posterior fascicle. ${ }^{3}$ Others have confirmed that this potential, thought to originate from Purkinje fibres in the posterior fascicle, is a good marker for the site of successful ablation. ${ }^{45} \mathrm{~A}$ similar electrogram was recorded from the site of successful ablation in the patients in this paper whose tachycardia arose from the anterior fascicle.

As has been reported, though pace mapping may be a useful guide as to the site for ablation, a perfect pace map is not essential for success. ${ }^{34}$

Ohe et al investigated four patients with right axis deviation during fascicular tachycardia. ${ }^{9}$ They found the earliest site of ventricular activation to be at the superior or superioseptal region of the left ventricle. Ablation was not carried out. Wen et al in their series of radiofrequency ablations of idiopathic left ventricular tachycardia mention one case of successful ablation of anterior fascicular tachycardia: the ablation site was in the anterior lateral wall of the left ventricle but no electrograms or other details were provided. ${ }^{4}$ Radiofrequency ablation of anterior fascicular tachycardia in this paper appears to be the second detailed report. The site of successful ablation was found in the anterior lateral wall 
inferior to the aortic valve and mitral valve ring, as in the case reported by Bogun et al. ${ }^{6}$

The appearance of lead V1 during tachycardia is widely described as a right bundle branch block configuration. ${ }^{1}$ However, there was in fact notable variation in appearance of V1 between patients and only two patients had classic right bundle branch block.

The success of radiofrequency ablation in this report compares favourably with other series: Nakagawa et al (seven of eight cases), ${ }^{3}$ Gonzalez et al (two of five cases), ${ }^{2}$ Wen et al (17 of 20 cases), ${ }^{4}$ and Katritsis et al (two of two cases). ${ }^{5}$

1 Ohe T. Idiopapthic verapamil-sensitive sustained left ventricular tachycardia. Clin Cardiol 1993;16:139-41.

2 Gonzalez R, Scheinman M, Lesh M, Helmy I, Torres V, Hare G. Clinical and electrophysiologic spectrum of fascicular tachycardias. Am Heart $\mathcal{F}$ 1994;128:147-56.
3 Nakagawa H, Beckman $\mathrm{K}$, McClelland J, Wang X, Arruda $\mathrm{M}$, Santoro I, et al. Radiofrequency catheter ablation of diopathic left ventricular tachycardia guided by a Purkinje potential. Circulation 1993;88:2607-17

4 Wen M, Yeh S, Wang C, Lin F, Chen I, Wu D. Radiofrequency ablation therapy in idiopathic left ventricular tachycardia with no obvious structural heart disease. Circulation 1994;89:1690-6.

5 Katritsis D, Heald S, Ahsan A, Anderson M, Camm J, Ward D, et al. Catheter ablation for successful management of left posterior fascicular tachycardia: an approach guided by recording of fascicular potentials. Heart 1996; 75:384-8.

6 Bogun F, El-Atassi R, Daoud E, Man K, Strickberger S, Morady F. Radiofrequency ablation of idiopathic left anterior fascicular tachycardia. F Cardiovasc Electrophysiol $1995 ; 6: 1113-6$.

7 Ward DE, Nathan AW, Camm AJ. Fascicular tachycardia sensitive to calcium antagonists. Eur Heart $f 1984 ; 5$ 896-905.

8 Kottkamp H, Chen $X$, Hindricks G, Willems S, Haverkamp $\mathrm{K}$, Wichter $\mathrm{T}$, et al. Idiopathic left ventricular tachycardia: new insights into electrophysiological characteristics and radiofrequency catheter ablation. Pacing Clin Electrophysiol 1995;18:1285-97.

9 Ohe T, Aihara N, Kamakura S, Kurita T, Shimizu W, Shimomura $\mathrm{K}$. Long-term outcome of verapamil-sensitive sustained left ventricular tachycardia in patients without structural heart disease. $\not{f} \mathrm{Am}$ Coll Cardiol 1995;25:54-8. 